\section{Kidney \\ Blood Pressure Research}

\title{
Downregulation of miR-503 in Activated Kidney Fibroblasts Disinhibits KCNN4 in an in Vitro Model of Kidney Fibrosis
}

\author{
Christoph Mann ${ }^{\mathrm{a}, \mathrm{b}} \quad$ Brajesh Pratap Kaistha ${ }^{\mathrm{a}} \quad$ Michael Kacika,c $^{\mathrm{a}} \quad$ Thorsten Stiewe $^{\mathrm{d}}$ \\ Joachim Hoyer ${ }^{\mathrm{a}}$ \\ aDepartment of Internal Medicine and Nephrology, University Hospital Marburg, Philipps-University, \\ Marburg, bepartment of Internal Medicine and Hematology, Oncology and Immunology, University \\ Hospital Marburg, Philipps-University, Marburg, 'Clemenshospital GmbH/Academic Teaching Hospital \\ of the Westphalian Wilhelms University of Muenster, Muenster, ${ }^{\mathrm{d} G e n o m i c s}$ Core Facility and Institute of \\ Molecular Oncology, Philipps-University, Marburg, Germany
}

\section{Key Words}

Kcnn4 • Kidney Fibrosis $・$ miRNA $・$ Ion Channel

\begin{abstract}
Background/Aims: Activated fibroblasts are key controllers of extracellular matrix turnover in kidney fibrosis, the pathophysiological end stage of chronic kidney disease. The proliferation of activated fibroblasts depends on the expression of the calcium-dependent potassium channel KCNN4. Expression of this ion channel is upregulated in fibrotic kidneys. Genetic and pharmacological blockade of KCNN4 inhibits fibrosis in vitro and in vivo. Methods: We studied the regulation of KCNN4 and possible involvement of miRNAs in an in-vitro fibrosis model using murine kidney fibroblasts. We tested fibroblast proliferation, channel function, channel expression and expression regulation after FGF-2 stimulation. Results: Proliferation was significantly increased by FGF-2, channel current and expression were almost doubled (+ $91 \%$ and $+125 \%$, respectively). MiRNA microarray identified upregulation of miRNA-503, which targets RAF1 and thereby controls KCNN4-expression via disinhibition of the Ras/Raf/MEK/ ERK-cascade. Conclusion: This data show a) a profound upregulation of KCNN4 in stimulated fibroblast and b) identifies miR-503 as a regulator of KCNN4 expression.
\end{abstract}




\section{Kidney Blood Pressure Research}

\section{Introduction}

Chronic kidney disease is an evolving global health problem affecting around $10 \%$ of the population. Regardless the different underlying causes of chronic kidney disease a rising fraction of patients progresses to end-stage renal-disease and requires renal replacement therapy [1]. The pathophysiological mechanism underlying this process is the replacement of functional parenchyma by extracellular matrix (ECM) resulting in kidney fibrosis and loss of organ function. This process depends on activated fibroblasts, the key controllers of ECMturnover. The proliferation of fibroblasts is driven by secretion of profibrotic cytokines like TGF- $\beta$ and FGF-2 [2] and depends on intracellular signal-transduction processes resulting in an increased intracellular calcium-concentration [3]. To maintain hyperpolarization of the plasma membrane during calcium influx fibroblasts - like in many other cells - express calcium-dependent potassium-channels in the cell membrane. These channels are activated by rising intracellular calcium concentrations and open for a compensatory efflux of potassium cations. Kidney fibroblasts rely on the calcium-dependent potassium-channel KCNN4 (syn. $\mathrm{K}_{\mathrm{Ca}} 3.1 ; \mathrm{IK}_{\mathrm{CA}} 1$ ). The expression of this ion channel is upregulated in activated fibroblasts. Its pharmacological blockade or genetic knockout impairs fibroblast proliferation and protects mice from UUO-induced kidney fibrosis [4]. One signal-transduction pathway that could be linked to the expression of KCNN4 is the Ras-Raf-MEK-ERK-Cascade. However, the details of this process are poorly understood [5]. This study aims to answer the question, whether micro-RNAs (miRNAs) play a role in the regulation of KCNN4-expression. MiRNAs are short, noncoding RNA-oligonucleotides of 21-25 bp length. They are expressed in a time- and tissue-specific manner and have been shown to impact many cellular processes including development, differentiation and proliferation $[6,7]$. They repress gene expression by binding to complementary regions in the 3-UTR of target-RNAs using a ribonucleoproteincomplex called RNA-induced silencing complex (RISC). This leads to direct cleavage, translational repression or mRNA-destabilization and impairs protein expression. To our knowledge, this is the first study testing their influence on ion channel expression and the regulation of KCNN4 in activated fibroblasts.

\section{Materials and Methods}

\section{Cell Culture}

A murine cell line of tubulointerstital kidney fibroblasts (TFBs) [8] was cultured in DMEM high Glucose $4.5 \mathrm{~g} / \mathrm{L}$ incl. Sodiumpyruvat + L-Glutamine $+5 \% \mathrm{FBS}+2 \%$ Penicillin/Streptomycin at $37^{\circ} \mathrm{C}$ in $5 \% \mathrm{CO}_{2}$ atmosphere. Cells were passaged at $80 \%$ confluence. Before stimulation, cells were starved for at least $72 \mathrm{~h}$ on DMEM high Glucose 4, 5g/L incl. Sodiumpyruvat + L-Glutamine + 0.5\% FBS + 2\% Penicillin/Streptomycin (starvation medium).

\section{Mitogenic stimulation}

Starved fibroblasts were trypsinized and 100, 000 cells were seeded in $10 \mathrm{ml}$ of starvation medium in petri dishes. After $24 \mathrm{~h}$ of settlement, cells were repetitively stimulated at $\mathrm{t} 1_{24 \mathrm{~h}}$ and $\mathrm{t} 2_{48 \mathrm{~h}}$ with FGF- 2 resulting in a final concentration of $10 \mathrm{ng} / \mathrm{ml}$ or vehicle (PBS-/-). At $\mathrm{t} 3_{72 \mathrm{~h}}$ cells were harvested for isolation of RNA, miRNA or seeded to small petri dishes on glass flasks for patch clamp.

\section{RNA Isolation \& cDNA Synthesis}

Cells were harvested after $72 \mathrm{~h}$. Total RNA was isolated using the glass-fibre column based high-purity RNAisolation Kit (Roche) following the manufacturers protocol including DNAse treatment. RNA was eluated in water and subjected to cDNA synthesis or stored at $-80^{\circ} \mathrm{C}$. cDNA was synthesized with anchored oligos using a Revert Aid $^{\mathrm{TM}}$ reverse transcriptase. cDNA libraries were subjected to qPCR. 


\section{Kidney Blood Pressure Research}

Quantitative real-time PCR

qPCR was performed in sealed 96-well plates using a 7500 Real Time PCR System (Applied Biosystems, USA). The mastermix consisted of $2 \mathrm{ul}$ cDNA, $2 \mathrm{ul}$ forward primer, $2 \mathrm{ul}$ reverse primer, 4ul PCR-grade water and 10ul Absolute Blue QPCR SYBR Green low ROX Mix (Thermo Scientific, USA). GAPDH was used as internal control and 4 wells were used as non-target control. Every sample was spotted in quadruplicate and ran after a hot start $\left(15 \mathrm{~min} @ 95^{\circ} \mathrm{C}\right)$ for 40 cycles $\left(1 \mathrm{~min} @ 60^{\circ} \mathrm{C} / 15 \mathrm{sec} @ 95^{\circ} \mathrm{C}\right)$. The following primers were used: GAP for: 5'-CAATGAATACGGCTACAGCAA. GAP rev: 5'-AGGGAGATGCTCAGTGTTGG. KCCN4 for: 5'-CATCACATTCCTGACCATCG. KCNN4 rev: 5'-ACGTGCTTCTCTGCCTTGTT. Every PCR-product was subjected to quality control using agarosegel electrophoresis to demonstrate specifity of the amplicon. A good correlation of at least 3 wells of the quadruplicate was considered necessary for evaluation; otherwise the reaction was performed again.

\section{Patch Clamp}

For patch-clamp experiments cultured fibroblasts after stimulation were transferred to glass microslides reusing the stimulation medium. After 3 hours of adherence electrophysiological experiments were performed in the whole-cell configuration as reported before [4]. Membrane currents were recorded with an EPC-9 patch-clamp amplifier (HEKA) using voltage ramps (1,000 ms; -100 to100 mV).

\section{Proliferation Assay}

Proliferation experiments were carried out in 96well plates using 1000 TFBs /well. Cells were cultivated in starvation media, stimulated after 24 and $48 \mathrm{~h}$ with FGF-2 1ng/100ul or PBS (controlgroup). After 72h Formazan was added and proliferation was measured using the MTT Cell Proliferation KIT (Roche, Switzerland) following manufacturers standard procedures. Absorption $\left(A_{550 \mathrm{~nm}}-A_{690 \mathrm{~nm}}\right)$ was measured using the EIA Reader Multiscan EX (Thermo Scientific, USA).

\section{MicroRNA Microarray}

Total RNA was labeled using the miRNA Complete Labeling and Hyb Kit (Agilent, USA). Samples were hybridized to the HD Mouse miRNA Microarray Kit (Agilent, Santa Clara, CA) according to the manufacturer's protocol. Arrays were scanned on the DNA Microarray Scanner G2505C (Agilent).

\section{Micro-RNA specific real-time PCR}

miRNA specific real-time PCR was carried out on a 7500 Real Time PCR System (Applied Biosystems, USA) using miRNA-specific stem-loop primers with FAM-Reporters detecting the target sequence of mmumiR-503 (UAGCAGCGGGAACAGUACUGCAG) (Roche, Assay ID: 002456). Stem-loop primers detecting snoRNA202 (GCTGTACTGACTTGATGAAAGTACTTTTGAACCCTTTTCCATCTGATG) were used as internal control (Roche, Assay ID: 001232). For reverse transcription TaqMan® MicroRNA Reverse Transcription Kit (Applied Biosystems,USA 4366596) was used. The reactions mastermix consisted of 0.15ul dNTPs; $1.0 u$ u MultiScribe $^{\mathrm{TM}}$ Reverse Transkriptase $(50 \mathrm{U} / \mu \mathrm{l}) ; 0.19 \mathrm{ul}$ RNase Inhibitor $(20 \mathrm{U} / \mu \mathrm{l}): 1.5 \mathrm{ul}$ reactionbuffer and TaqMan ${ }^{\circledR}$ MicroRNA Assays (Applied Biosystems, USA) were used.

\section{Results}

Upregulation of the KCNN4 expression and an increase in fibroblast proliferation were considered as necessary features to demonstrate the functionality of the used in-vitro fibrosis model.

Expression of the KCNN4 channel was evaluated on mRNA level using qPCR and on the level of protein function using patch clamp. After mitogenic stimulation with FGF-2 (10ng/ $\mathrm{ml}$ ) we saw a robust 2.3-fold upregulation of KCNN4-mRNA compared to control in qPCR (Fig. 1). Channel activity representing protein function increased 1.9 -fold at $0 \mathrm{mV}$ measured by patch-clamp (Fig. 2 \& Fig. 3). The specificity of the cation current was demonstrated by its nearly complete suppression after addition of the KCNN4-specific blocker TRAM34 excluding significant contribution of other $\mathrm{K}_{\mathrm{Ca}}$-channels to this current. Experiments 


\section{Kidney Blood Pressure Research}

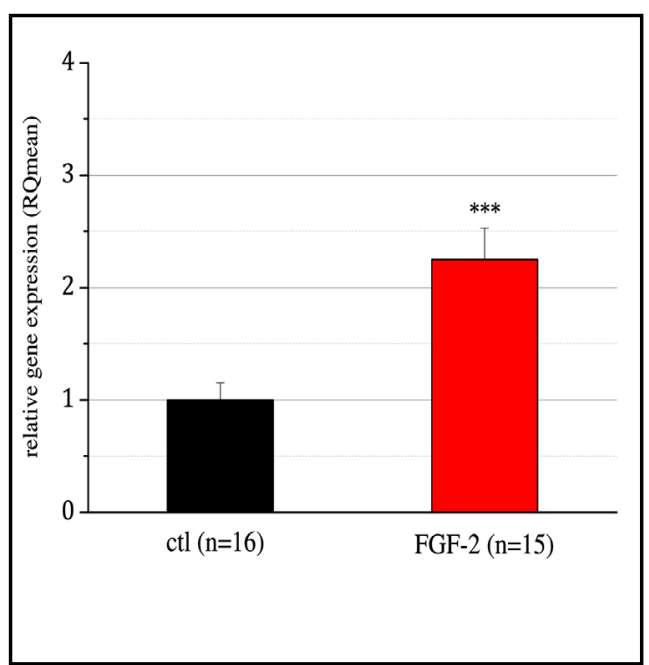

Fig. 1. Mean relative gene expression of KCNN4 after qPCR.

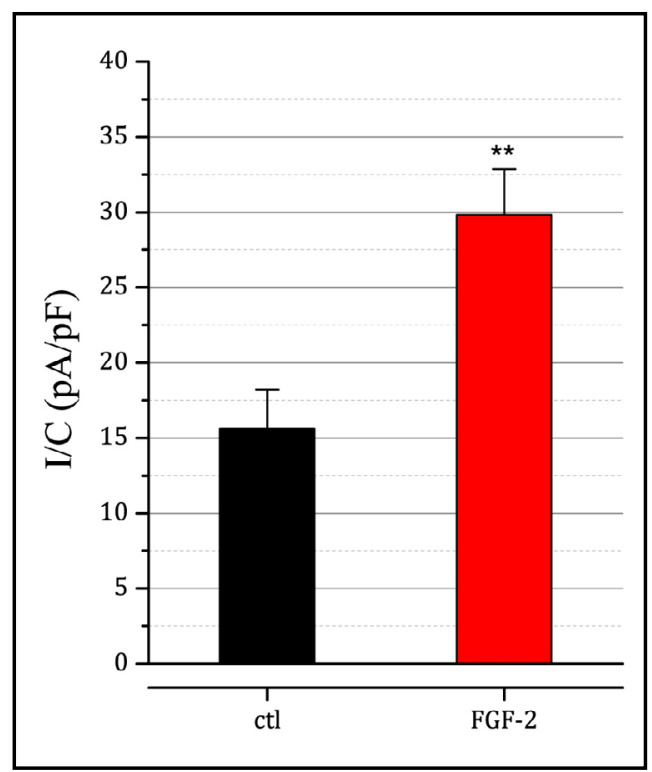

Fig. 2. Mean KCNN4-specific current @0mv measured in whole-cell patch clamp analyses displayed in relative capacity I/C.

Fig. 3. Mean KCNN4-specific current @0mv measured in whole-cell patch clamp analyses displayed in relative capacity I/C.

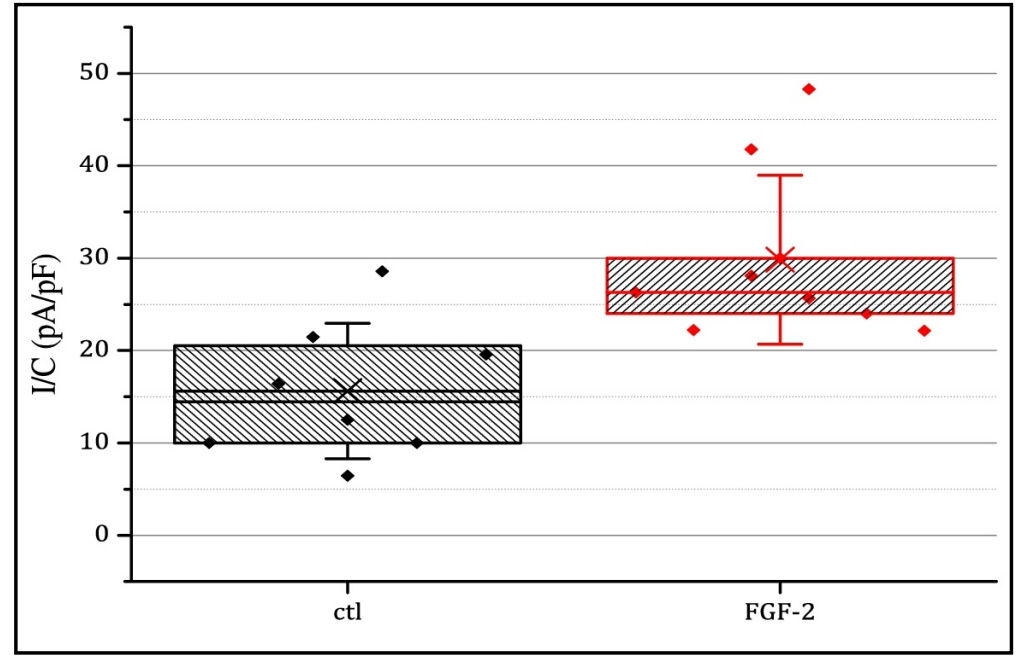

were performed in a blinded mode leaving the patch clamp investigator uninformed of the preceding treatment. The proliferation assay was based on standard MTT-based methodology [9]. The reduction of MTT to Formazan, a reaction depending on NADPH regeneration of metabolic active cells, was quantified by measurement of absorbance $\left(A_{550 \mathrm{~nm}}-A_{690 \mathrm{~nm}}\right)$. The FGF-2 group ( $n=131$ wells) showed higher absorbance compared to placebo $(p<0,001)$, which extends the range of 1 standard deviation (Fig. 4A and B). This verified the mitogen potential of FGF-2 in this setting. After demonstrating the liability of this model in inducing proliferation and ion channel upregulation, a miRNAscreen was performed. The miRNAexpression in two cohorts of fibroblasts, after stimulation with FGF-2 or with placebo, was compared using a miRNA micro array. A miRNA was considered to be differentially regulated as the expression between both groups differed by a factor of at least 2 ( $\mathrm{fc} \geq 2$ ) (Fig. 5, Fig. 6, and Table 1). All differentially regulated miRNAs were subjected to in-silico target prediction analysis for KCNN4 using Target Scan [11]. No miRNA was predicted to directly target KCNN4 -3'UTR. However, miR-503, the miRNA with the highest estimated foldchange in the 


\section{Kidney \\ Blood Pressure Research}

screening array was predicted to target RAF1, a known upstream regulator of KCNN4 via the Ras-Raf-MEK-ERK cascade [5]. Therefore, miR-503 was picked for further evaluation. A miRNA specific quantitative rt-PCR was used to validate the expression change after FGF2 stimulation. The qPCR of 4 samples of each group (FGF-2 vs. Ctl) confirmed significant downregulation of miR-503 by FGF-2 stimulation (Fig. 7). Further, analysis of predicted miR-503s binding site at nucleotide 108-114 of RAF1-3'UTR was conducted. To demonstrate relevant suppression of RAF1 by miR-503 we used the following criteria implemented in the context+-score of the TargetScanS algorithm [6, 11-14]. The seed region consists of a highly conserved 7 mer1Abinding site (site-type contribution: -0.074. $\mathrm{p}_{\mathrm{Ct}}=0.85$. rank 55 of 385.). Also, site context (context+ score $-0.17 ; 60$. percentile), target site abundance (TA contribution: -0.019), and seed-pairing stability (SPS contribution: -0.066) predict a high probability of biologically relevant suppression of RAF1 at this binding site. The possible additional 3'-supplementary pairing at position 1417 and 19 seems unlikely since this part of the RAF1-UTR is not conserved (Fig. 8).

\section{Discussion}

The established in vitro fibrosis model demonstrates a mitogenic effect of FGF-2 on kidney fibroblasts. This is expanding knowledge to already published and unpublished data generated by our group [4] and others [15]. The size of the effect in our model is smaller than published before in a similar setting [4]. This is likely to be due to differences in the used MTTassay based on metabolism in comparison to the available data based on BrDU assays relying on DNA synthesis. Proliferating cells including activated fibroblasts rely on increased $\mathrm{K}^{+}-$ membrane-conductivity through KCNN4 channels. A widely accepted hypothesis for this is to maintain membrane hyperpolarization for sustained calcium influx by increased potassium efflux through calcium-activated potassium channels. This study approves this model by demonstrating a highly significant increase not only in KCNN4-mRNA but also in increased channel-function in whole-cell patch clamp analysis. Of note, the nearly complete suppression of the current by the selective blocker TRAM-34 excludes significant contribution of other $\mathrm{K}_{\mathrm{Ca}}$-channels than KCNN4 to this current. This is strengthened by the good correlation of

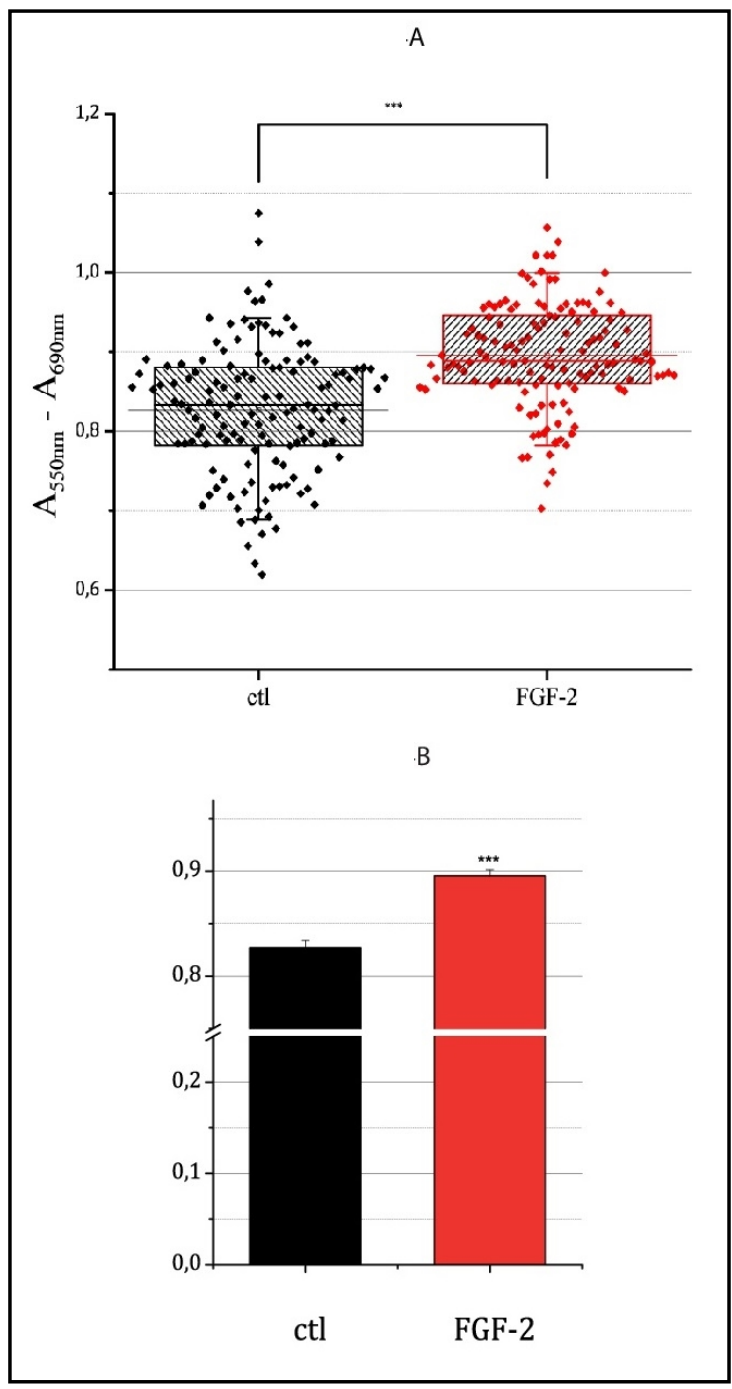

Fig. 4. Absorbance in MTT proliferation assay comparing control $(\mathrm{n}=132)$ and FGF2 group (red, $\mathrm{n}=131$ ) after mitogenic stimulation or placebo. One outlier >1.5 in FGF Group was considered pipetting error and excluded. (A) bow plot: box ranges from 25 th to 75 th percentile. Line in the middle: mean. Square in the middle: median. Whisker: +/- 1 standard devation (SD). (B) Mean Absorbance in the FGF-2 Group is increased by $7 \%(\mathrm{p}<0,001)$.Whisker: +/- SEM. Note: $\mathrm{y}$-axis is hidden $0,25-0,75$. 


\section{Kidney Blood Pressure Research}

Fig. 5. Of 651 spotted miRNAs (miRBase 12.0; [10]) 403 showed a relevant signal. Of these, 22 fulfilled the criteria of being differentially regulated. 15 miRNAs showed significant upregulation after FGF2 stimulation, 7 were downregulated.

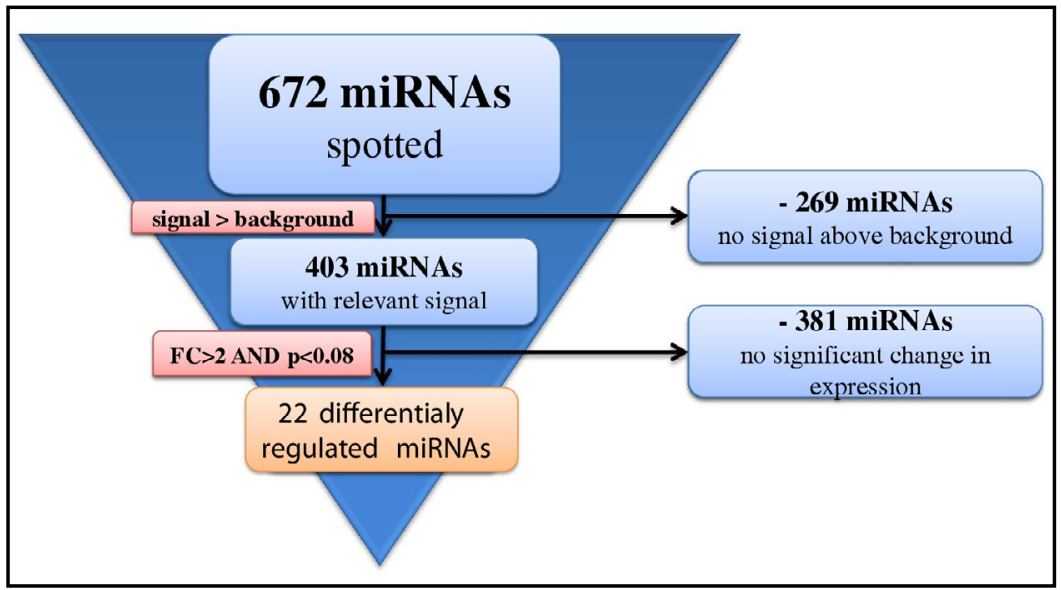

Fig. 6. Regulation of miRNA expression after FGF-2 stimulation, displayed as $\log 2$ of the foldchange of control to FGF-2 group by miRNA micro array. Marked in blue: the 22 differentially regulated miRNAs (see Table 1). Marked in red: miRNA with the highest foldchange after stimulation: miR-503 (fc $=-4.19 . p=0.34)$.

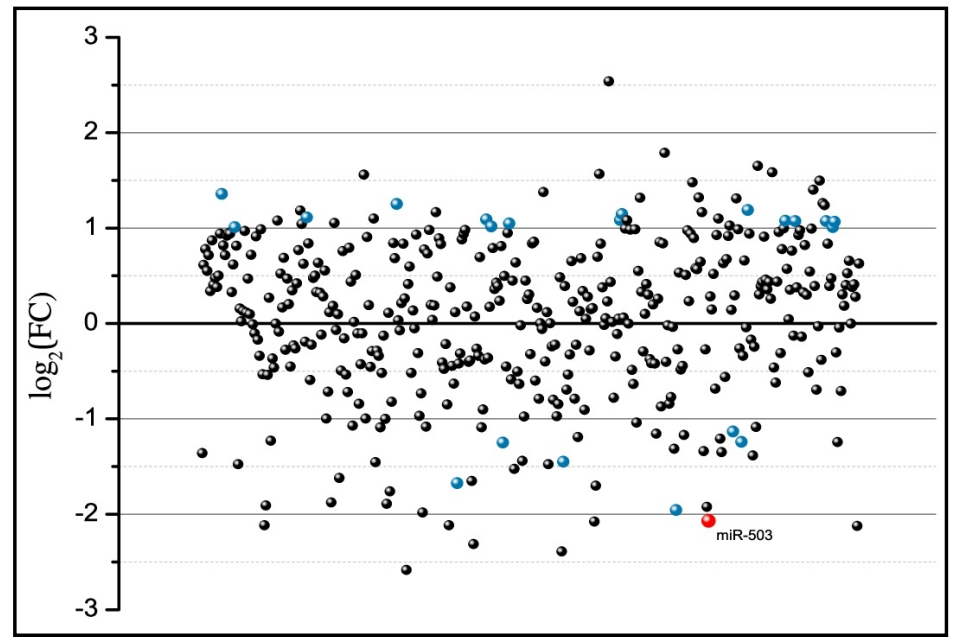

the foldchange in qPCR $(\mathrm{fc}=+2.25)$ and patch clamp $(\mathrm{fc}=+1.91)$. To proceed to the key question of miRNAs influencing this process of upregulation, the insilico target prediction of differentially regulated miRNAs showed no binding site in KCNN4-3'UTR, but a binding site in RAF1-3'UTR suggests indirect regulation of KCNN4 by miR-503 targeting this upstream regulator. The bioinformatical analysis of the binding site considering conserved seed pairing [11] and site context [13] revealed a high probability of biological active suppression auf RAF1 by miR-503. miR-503 has been classified as part of the extended miR-16-family [16]. It clusters with miR-424, which is located only $383 \mathrm{bp}$ next to miR-503 at X.q26.3. Considering the criteria target site abundance and seed-pairing stability published by Garcia et al. in 2011, miR-503 has $10^{3.296}$ predicted binding sites $\left(42^{\text {nd }}\right.$
Table 1. Differentially regulated miRNAs after miRNA microarray

\begin{tabular}{lclc}
\hline Identifier & p-Value & foldchange & regulation type \\
\hline mmu-miR-503 & 0.034 & -4.1896 & down \\
mmu-miR-467a* & 0.031 & -3.8762 & down \\
mmu-miR-219 & 0.013 & -3.1864 & down \\
mmu-miR-326 & 0.005 & -2.7268 & down \\
mcmv-miR-m108-1* & 0.029 & 2.5675 & up \\
mmu-miR-1903 & 0.006 & 2.3818 & up \\
mmu-miR-297a* & 0.011 & -2.3725 & down \\
mmu-miR-615-3p & 0.034 & -2.3648 & down \\
mmu-miR-667 & 0.007 & 2.2835 & up \\
mmu-miR-409-3p & 0.025 & 2.2152 & up \\
mmu-miR-582-5p & 0.005 & -2.1920 & down \\
mmu-miR-129-3p & 0.020 & 2.1635 & up \\
mmu-miR-290-3p & 0.012 & 2.1332 & up \\
mmu-miR-384-3p & 0.003 & 2.1202 & up \\
mmu-miR-702 & 0.013 & 2.1114 & up \\
mmu-miR-715 & 0.028 & 2.1079 & up \\
mmu-miR-877* & 0.014 & 2.1062 & up \\
mmu-miR-92b & 0.021 & 2.0958 & up \\
mmu-miR-299* & 0.014 & 2.0694 & up \\
mmu-miR-291b-5p & 0.023 & 2.0252 & up \\
mghv-miR-M1-7-3p & 0.033 & 2.0134 & up \\
mmu-miR-92a* & 0.027 & 2.0133 & up \\
\hline
\end{tabular}




\section{Kidney Blood Pressure Research}

Fig. 7. $\Delta \mathrm{Ct}$ of miR-503 in miRNAspecific quantitative real-time PCR is significantly higher in FGF-2 stimulated fibroblasts resulting in a reduction of relative miR503 gene expression down to $13 \%$ ( $\mathrm{fc}=-7.7 . \mathrm{p}<0.01$ ) compared to control confirming significant downregulation. 4 samples per group. Shown is $\Delta \mathrm{Ct}+/-\mathrm{SE}$ (RQmin/Max) calculated for every individual PCR-reaction.

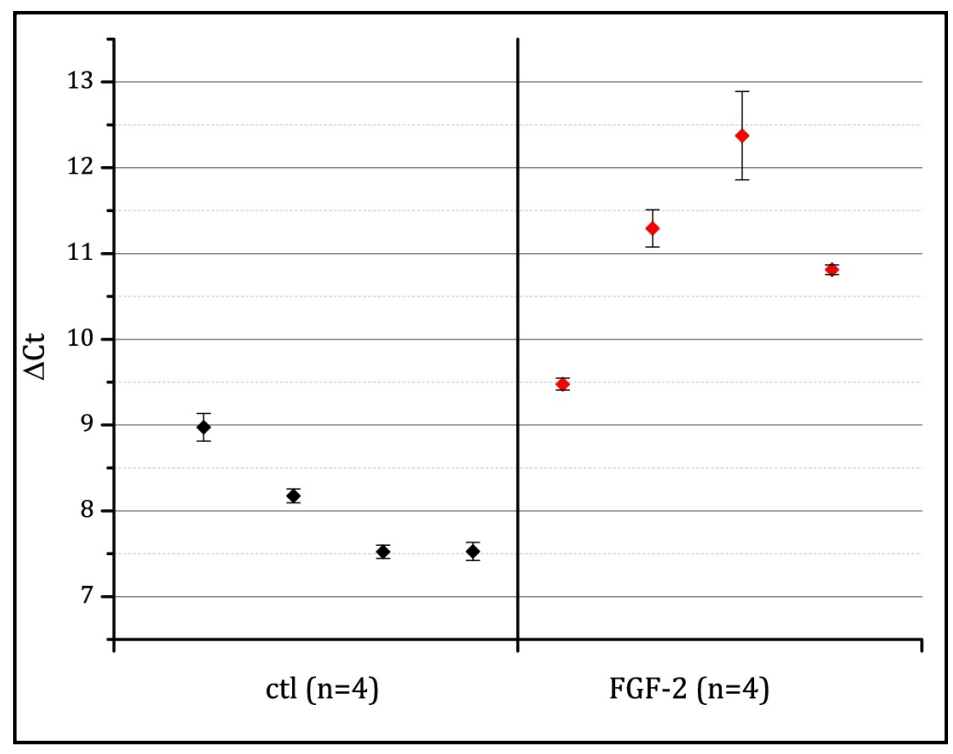

percentile), which is lower than the median number of binding sites $\left(10^{3.495}\right)$ and significantly lower than the number of binding sites of the other family members of the extended miR$15 / 16$ family $\left(10^{3.667}\right.$ predicted binding sites, $90^{\text {th }}$ percentile) [14]. The seed-pairing stability of miR-503 $(-10,93 \mathrm{kcal} / \mathrm{mol})$ is predicted to be significantly higher compared to the median of conserved, vertebrate miRNAs $(-7.87 \mathrm{kcal} / \mathrm{mol})$ and higher than the other family members $(-10.23 \mathrm{kcal} / \mathrm{mol})$. This suggests a specialized role of miR-503 in the extended miR-15/16 family suppressing precisely fewer target-genes with higher efficiency. Focusing on the ion channel regulation our study proposes a model of cooperation of growth-factor induced activation and parallel disinhibition of RAF1 through miR-503 downregulation leading to an increase in KCNN4 activity and enhancing fibroblast proliferation (Fig. 9). This makes miR-503 a relevant target for further research regarding diseases relying on increased cellproliferation like fibrosis or cancer.

\section{Conclusion}

This study confirms the mitogenic effect of FGF-2 on fibroblasts. It ensures the upregulation of the KCNN4 in stimulated fibroblasts and proposes miR-503 playing a significant role in this process by disinhibiting the Ras-Raf-MEK-ERK cascade. 
Kidney

Blood Pressure Research

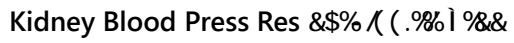

\begin{tabular}{|l|l|}
\hline DOI: 10.1159/000498875 & (c) 2019 The Author(s). Published by S. Karger AG, Basel \\
\hline
\end{tabular}

Published onlıne: 22 February 2019 www.karger.com/kbr

120

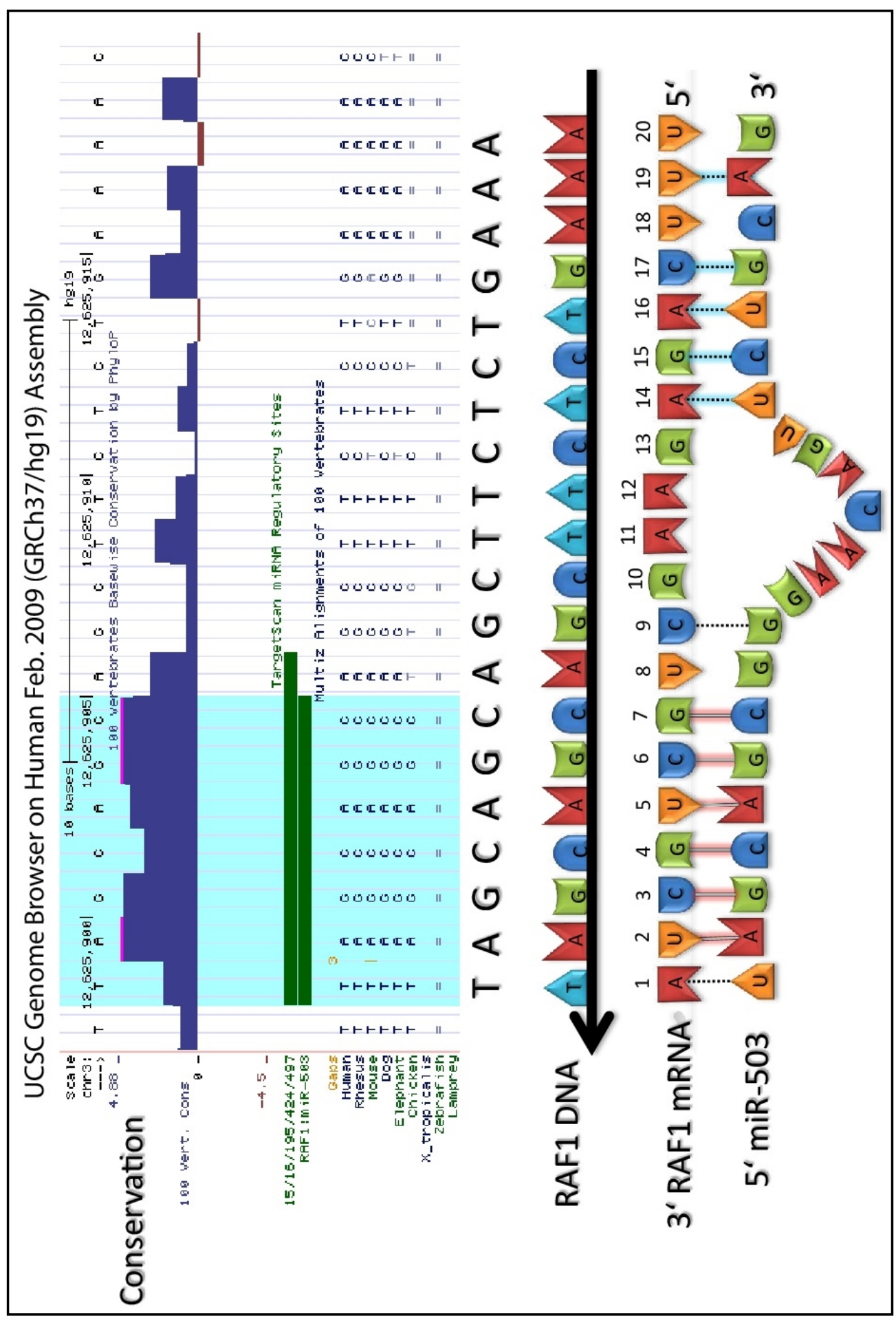

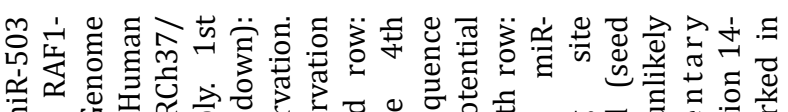
当.

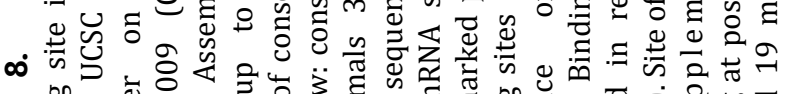

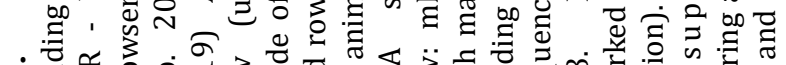

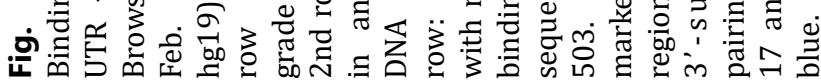




\section{Kidney Blood Pressure Research}

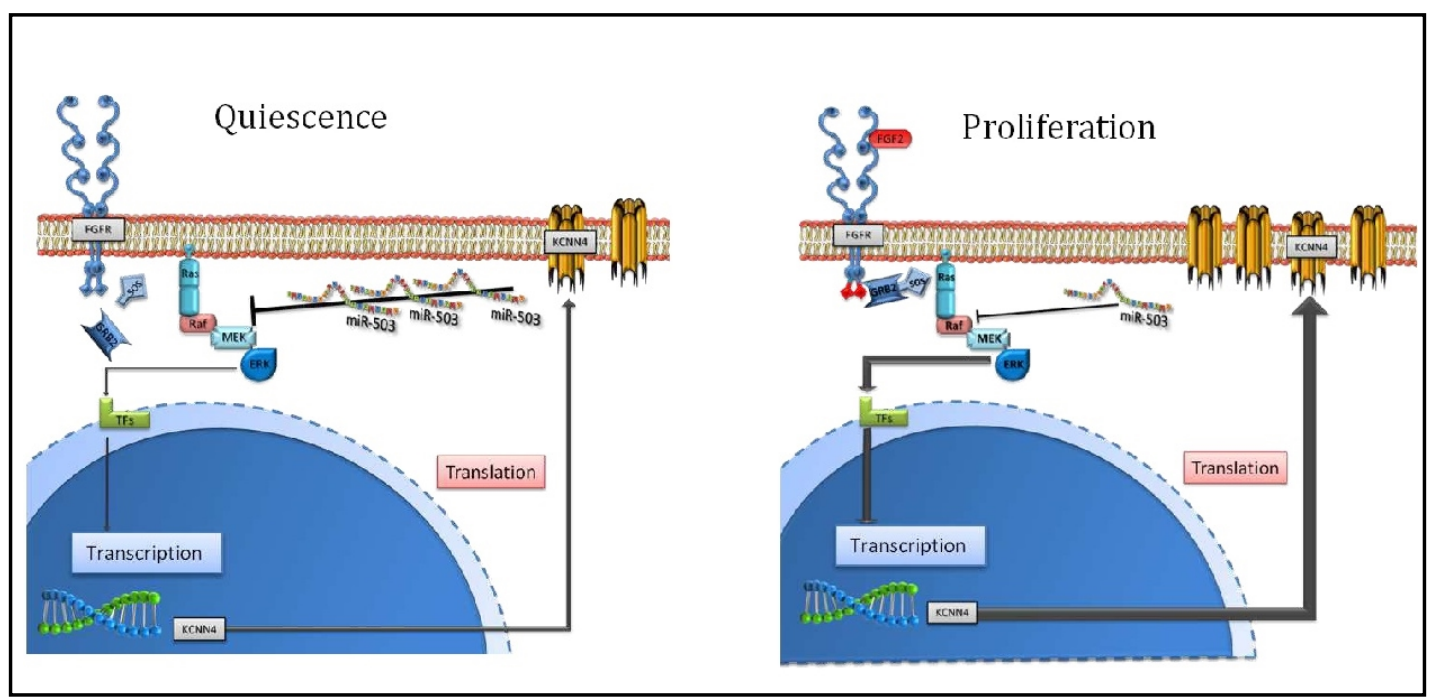

Fig. 9. Quiescence: The resting cell has low activity of Ras-Raf-MEK-ERK cascade due to low activity of the FGF-2-receptor and high concentration of inhibitory miR-503. Proliferation: After binding of growth-factors to FGF-2-R the cascade is activated upstream by GRB2 and SOS. Additionally the lower concentration of the inhibitory miR-503 disinhibits RAF1. The higher cascade-activity leads to an increased transcription of KCNN4 via transcription factors and results in increased translation of KCNN4-channels.

\section{Acknowledgements}

The Authors thank Felix Kurtz, Anuradha Kaistha and Sigrid Bischofsberger for their help in delivering this research.The authors have no ethical conflicts to disclose. JH received founding from the Deutsche Forschungsgemeinschaft: FOR-1086-2. CM, BPK and JH designed the research. CM performed the research. BPK supervised the research. MK performed the patch clamp analysis. TS hosted the micro-array in the genomics core facility. CM and BPK analysed the Data. CM and JH wrote the Paper.

\section{Disclosure Statement}

J.H. is inventor on a University of California patent claiming KCa3.1 blockers for the treatment of fibrosis.

\section{References}

1 Radhakrishnan J, Remuzzi G, Saran R, Williams DE, Rios-Burrows N, Powe N, Brück K, Wanner C, Stel VS, Venuthurupalli SK, Hoy WE, Healy HG, Salisbury A, Fassett RG, O'Donoghue D, Roderick P, Matsuo S, Hishida A, Imai E, Iimuro S: Taming the chronic kidney disease epidemic: a global view of surveillance efforts. Kidney Int 2014;86:246-250.

-2 Strutz F, Zeisberg M, Hemmerlein B, Sattler B, Hummel K, Becker V, Muller GA: Basic fibroblast growth factor expression is increased in human renal fibrogenesis and may mediate autocrine fibroblast proliferation. Kidney Int 2000;57:1521-1538.

3 Follonier Castella L, Gabbiani G, McCulloch CA, Hinz B: Regulation of myofibroblast activities: Calcium pulls some strings behind the scene. Exp Cell Res 2010;316:2390-2401.

4 Grgic I, Kiss E, Kaistha BP, Busch C, Kloss M, Sautter J, Müller A, Kaistha A, Schmidt C, Raman G, Wulff H, Strutz F, Gröne H-J, Köhler R, Hoyer J: Renal fibrosis is attenuated by targeted disruption of KCa3.1 potassium channels. Proc Natl Acad Sci 2009;106:14518-14523. 


\section{Kidney \\ Blood Pressure Research}

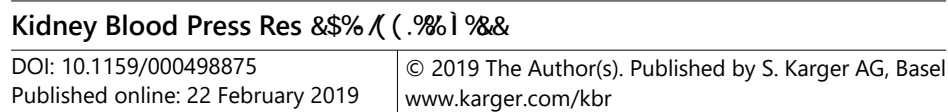

Mann et al.: Downregulation of miR-503 Disinhibits KCNN4 in Kidney Fibrosis

5 Peña TL, Chen SH, Konieczny SF, Rane SG: Ras/MEK/ERK Up-regulation of the Fibroblast KCaChannel FIK Is a Common Mechanism for Basic Fibroblast Growth Factor and Transforming Growth Factor- $\beta$ Suppression of Myogenesis. J Biol Chem 2000;275:13677-13682.

6 Lewis BP, Shih IH, Jones-Rhoades MW, Bartel DP, Burge CB: Prediction of Mammalian MicroRNA Targets. Cell 2003;115:787-798.

7 Bartel DP: MicroRNAs: genomics, biogenesis, mechanism, and function. Cell 2004;116:281-297.

-8 Alvarez RJ, Sun MJ, Haverty TP, Iozzo RV, Myers JC, Neilson EG: Biosynthetic and proliferative characteristics of tubulointerstitial fibroblasts probed with paracrine cytokines. Kidney Int 1992;41:14-23.

-9 Berridge MV, Tan AS: Characterization of the cellular reduction of 3-(4, 5-dimethylthiazol-2-yl)-2,5diphenyltetrazolium bromide (MTT): subcellular localization, substrate dependence, and involvement of mitochondrial electron transport in MTT reduction. Arch Biochem Biophys 1993;303:474-482.

10 Kozomara A, Griffiths-Jones S: miRBase: integrating microRNA annotation and deep-sequencing data. Nucleic Acids Res 2011;39:D152-D157.

11 Lewis BP, Burge CB, Bartel DP: Conserved seed pairing, often flanked by adenosines, indicates that thousands of human genes are microRNA targets. Cell 2005;120:15-20.

12 Whitehead Institute for Biomedical Research: TargetscanHuman 6.2: predicted miRNA targets of miR-503. URL: http://www.targetscan.org/cgi-bin/targetscan/vert_61/targetscan.cgi?species=Mouse\&gid=\&mir_ $\mathrm{sc}=$ miR-503\&mir_c=\&mir_nc=\&mirg=.

13 Grimson A, Farh KK-H, Johnston WK, Garrett-Engele P, Lim LP, Bartel DP: MicroRNA Targeting Specificity in Mammals: Determinants beyond Seed Pairing. Mol Cell 2007;27:91-105.

14 Garcia DM, Baek D, Shin C, Bell GW, Grimson A, Bartel DP: Weak seed-pairing stability and high target-site abundance decrease the proficiency of lsy- 6 and other microRNAs. Nat Struct Mol Biol 2011;18:1139-1146.

-15 Strutz F, Zeisberg M, Renziehausen A, Raschke B, Becker V, Van Kooten C, Muller GA: TGF- $\beta 1$ induces proliferation in human renal fibroblasts via induction of basic fibroblast growth factor (FGF-2). Kidney Int 2001;59:579-592.

16 Rissland OS, Hong S-J, Bartel DP: MicroRNA Destabilization Enables Dynamic Regulation of the miR-16 Family in Response to Cell Cycle Changes. Mol Cell 2011;43:993-1004. 